Einführung zum Thema

Pneumologe $2020 \cdot 17: 1-2$

https://doi.org/10.1007/s10405-019-00287-4

๑) Springer Medizin Verlag GmbH, ein Teil von Springer Nature 2019

Die interventionelle Pneumologie gehört zu den Teilgebieten der Lungenheilkunde, die in den letzten Jahren zu bedeutenden Änderungen der klinischen Abläufe geführt hat. Auf dem Boden technischer Weiterentwicklung und Miniaturisierung von Instrumenten wurde es möglich, diagnostische und therapeutische Prozesse minimal-invasiver und risikoärmer für die Patienten durchzuführen. Hierdurch konnten auch Patienten einen Zugang zu Therapien bekommen, denen es zuvor z.B. aufgrund von Komorbiditäten nicht möglich war.

Das therapeutische Ziel interventioneller Verfahren ist in aller Regel die Verbesserung oder Erhaltung der Lebensqualität und die Verminderung von Symptomen. Minimal-invasive Verfahren sind dabei nicht per se besser oder risikoärmer als invasivere Verfahren. Möglichkeiten und Grenzen der Methoden, aber auch der eigene Ausbildungsstand sollten dem interventionell tätigen Pneumologen bewusst sein. Nur unter dieser Prämisse können, bei präziser Indikationsstellung und sorgfältiger Durchführung, minimalinvasive Methoden zu einer Reduktion von Morbidität und Mortalität führen. Es darf nicht unerwähnt bleiben, dass das DRG-Abrechnungssystem die weitere Nutzung und Verbreitung interventioneller Verfahren nicht fördert, da ein weniger invasives Verfahren in der Regel schlechter vergütet wird, als ein invasives Verfahren. Es wäre auch im Sinne der Patienten wünschenswert, wenn diese Fehlanreize vermindert würden.

Dieses Übersichtsheft möchte einen aktuellen Überblick über verschiedene Methoden der interventionellen Pneumologie geben. Zu den wesentlichen Entwicklungen der letzten Jahre gehören der

\title{
K. Darwiche
}

Pneumologische Universitätsklinik, Ruhrlandklinik - Universitätsmedizin Essen, Essen, Deutschland

\section{Interventionelle Pneumologie und Bronchologie}

endobronchiale Ultraschall (EBUS) mit seinen beiden meistgenutzten Modalitäten EBUS-TBNA (endobronchialer Ultraschall - transbronchiale Nadelaspiration) und radiärer Ultraschall („Minisonde") sowie die interventionelle Emphysemtherapie. $\mathrm{Zu}$ den neueren Entwicklungen gehört die navigierte Bronchoskopie und die bronchoskopische Behandlung der nicht vorwiegend emphysematösen chronisch obstruktiven Lungenerkrankung (COPD). Weitere relevante Verfahren (z.B. die transbronchiale Kryobiopsie) konnten aus Kapazitätsgründen nicht in diesem Heft aufgeführt werden.

Der Auftakt in diesem Heft gebührt dem „Altmeister der interventionellen Pneumologie“: der bronchoskopischen Behandlung von Stenosen der zentralen Atemwege mit thermischen Verfahren oder durch Überbrückung mit Stents. Diese klassischen Methoden der starren Bronchoskopie sind seit Jahrzehnten etabliert. In neuerer Zeit konnten allerdings auch hier Verbesserungen, z. B. durch die Entwicklung neuer Stents erreicht werden. L. Freitag(Luzern/Hemer/ Essen) beschreibt, welches interventionell-therapeutische Vorgehen auf Basis von Genese und Morphologie der Stenose $\mathrm{zu}$ bevorzugen ist. Die klinische Erfahrung, die notwendig ist, um das optimale Resultat für den betroffenen Patienten zu erreichen, wird nicht selten unterschätzt.

In den letzten 15 Jahren wurden mehrere bronchoskopische Verfahren zur interventionellen Emphysemtherapie entwickelt, zu denen die Ventil- oder Coilimplantation und die Behandlung mit Wasserdampf oder Polymerschaum gehören. Die Selektion der für eine Therapie in Frage kommenden Patienten ist der
Schlüssel des Therapieerfolgs. Jedes dieser Verfahren hat, orientierend an Ausprägung und Verteilung des Emphysems sowie dem Vorhandensein oder Fehlen einer kollateralen Ventilation eine leicht unterschiedliche Indikation. Hieraus ergibt sich, dass die optimale Behandlung der im Alltag schwer eingeschränkten Patienten in einem Zentrum stattfinden sollte, in dem möglichst alle diese Verfahren durchgeführt werden. Ralf-Harto Hübner (Berlin) leitet eines dieser Zentren und stellt den aktuellen Stand des Wissens dar.

M. Schumann (Konstanz) stellt in ihrem Beitrag die aktuellen interventionellen Therapiemöglichkeiten bei chronisch-obstruktiven Atemwegserkrankungen dar. Dies umfasst einerseits die bronchiale Thermoplastie zur Behandlung des chronisch refraktären Asthmas und andererseits die Möglichkeiten, durch einen endoskopischen Eingriff den Verlauf und die Symptomatik bei Patienten mit schwerer COPD, bei denen ein Emphysem nicht im Vordergrund steht, positiv zu beeinflussen. Wer wissen will, ob und wie dies gelingen kann, wird in dem Artikel Antworten finden.

Die Aussage, dass der EBUS eine der wesentlichen Entwicklungen der Pneumologie der letzten Jahre darstellt, ist keine Übertreibung. Auf dem Boden hoher Evidenz ist der EBUS als zentraler Baustein in der Diagnostik des Lungenkarzinoms klinisch etabliert. Es gibt spannende Weiterentwicklungen, die von $S$. Eisenmann (Halle/Saale) ausführlich dargestellt werden. Der Artikel offenbart, dass das Potenzial der Methode noch nicht ausgeschöpft worden ist.

Eigentlich ist der Rundherd ein „alter Hut“. Allerdings repräsentiert gerade dieses Thema die Moderne der interven- 
tionellen Pneumologie. Digitalisierung, augmentierte und virtuelle Realität sowie Robotik haben die Rate erreichbarer und diagnostizierbarer peripherer Herde deutlich gesteigert und werden diese in der näheren Zukunft noch weiter verbessern. J. Winantea (Essen) beleuchtet hierbei auch die zukünftige Möglichkeit der bronchoskopisch geführten Ablation peripherer Lungenherde.

Dieses Heft gibt Ihnen die Gelegenheit, sich über verschiedene Aspekte des innovativen Teilgebiets der interventionellen Pneumologie zu informieren. Ich wünsche Ihnen viel Spaß beim Lesen.

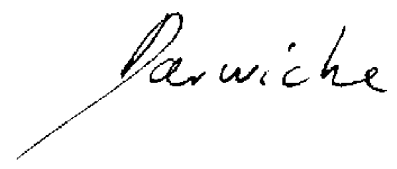

PD Dr. Kaid Darwiche

\section{Korrespondenzadresse}

\section{PD Dr. K. Darwiche}

Pneumologische Universitätsklinik, Ruhrlandklinik - Universitätsmedizin Essen Tüschener Weg 40, 45239 Essen, Deutschland kaid.darwiche@rlk.uk-essen.de

Interessenkonflikt. K. Darwiche: Honorare aus Referententätigkeit, für Beratertätigkeit und Forschungsunterstützung: Olympus, Boston Scientific, Broncus Medical, Erbe, Böhringer Ingelheim, bess, Storz, Pulmonx, PneumRx, Nuveira, Epigenomics, Novartis, Roche.

\section{Dank an die Gutachter*innen}

Für die Qualität und Objektivität der Beiträge sind neben den engagierten Autorinnen und Autoren auch die vielen qualifizierten Gutachterinnen und Gutachter maßgeblich, die im Rahmen des Peer-Review-Prozesses die Manuskripte inhaltlich-wissenschaftlich prüfen und Empfehlungen zur konkreten Verbesserung äußern. Allen Gutachterinnen und Gutachtern, die im vergangenen Jahr Manuskripte für diese Zeitschrift begutachtet haben, danken wir herzlich für die konstruktive und gewissenhafte Arbeit.

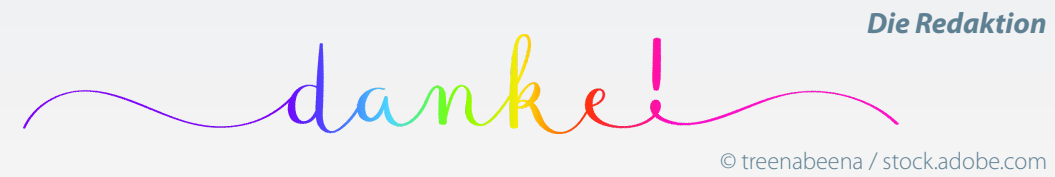

